\title{
El soplador de vidrio
}

\author{
Justina Rodríguez García*
}

\begin{abstract}
RESUMEN ABSTRACT
Se analiza, en primer lugar, la figura del artista del vidrio de Murano durante los siglos XV al XVII y la importancia y repercusión de su obra en toda Europa, para pasar después a estudiar la actividad del vidriero catalán y castellano, muy condicionada por la moda procedente de Venecia, asi como la distinta manera de hacer frente a esta dura competencia por parte de los artesanos de ambas zonas de España.

At first the Murano's glass artist's figure during the fifteenth to seventeenth centuries and the importance and repercussion of his creation in whole Europe is analysed. After this the paper study $y$ treats of the Catalan and Castillian glassblowers's activity, which was very conditioned by the Venetian mode, as well as the different way showed by those mentioned spanish craftsmen of facing this hard competence.
\end{abstract}

El arte del vidrio, que de tan alta estima gozó en las cortes europeas de los tiempos modernos, debido a su importancia como elemento surituario indispensable en los ajuares y decoración de los palacios de las elites sociales, tuvo como artífices a personajes que, por lo común, formaban parte de conocidas familias dedicadas al oficio durante generaciones, especialmente en los más importantes centros productores de vidrio artístico, como Venecia y Bohemia. Las dificultades técnicas que llevaba im-

\footnotetext{
* Departamento de Historia Medieval y Moderna. UNED.
} 
plícita la tarea del vidriero, una de cuyas principales metas era la investigación de nuevas fórmulas que aportaran una constante creatividad y, en consecuencia, un mayor prestigio a su producción, determinaron la transmisión del oficio de padres a hijos, con el especial compromiso de custodiar celosamente los hallazgos técnicos alcanzados - los llamados "secretos", de los que aún en nuestros dias siguen vanagloriándose las más importantes fábricas-.

Me estoy refiriendo, como es obvio, al vidriero dedicado a la manufactura de vidrio suntuario, en cuya labor existía una clara intención artística, ya que el encargado de soplar el llamado vidrio común era un artesano de escaso relieve social, que atendía una demanda de primera necesidad.

Voy a centrarme, en primer lugar, en el vidriero de Murano, por ser esta isla italiana el centro productor de vidrio suntuario más importante durante los siglos XV al XVII, llegando a ejercer enorme influencia en el resto de Europa. A continuación, y sobre esta base comparativa, me referiré al vidriero español, y más concretamente, al catalán y al castellano, que durante estas mismas centurias vieron su actividad profesional muy mediatizada por la competencia de los muraneses.

Esta difusión de la producción de los hornos de Murano fuera de las fronteras de la República de Venecia es el fenómeno conocido por los his-

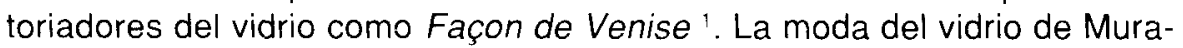
no se impuso de tal manera que la comercialización de las piezas no fue suficiente para hacer frente a la importante demanda. Simultáneamente, tuvo lugar la evasión de numerosos vidrieros muraneses que se establecieron en las diversas capitales europeas, en donde se les reclamaba para trabajar y enseñar a los artesanos locales, intentando los gobiernos de los distintos países, a través de este sistema, abaratar costos y atender más ágilmente las exigencias del mercado.

Como en todo trabajo artesanal, la reglamentación laboral en Murano estaba perfectamente regulada a través de las asociaciones gremiales. La Mariegola dell'Arte dei verieri di Murano, cuyos orígenes se remontan a la primera mitad del siglo $x v$, recoge todas las disposiciones y normas que debian regir la actividad de los operarios del vidrio, puestas en práctica bajo la celosa vigilancia del Estado, así como la tasación de los ob-

La expresión se aplica al fenómeno de expansión del vidrio de Murano por Europa a través de los artifices evadidos y de la exportación, y también a un estilo decorativo propio de los cbjetos de vidrio elaborados a la manera de Venecia. 


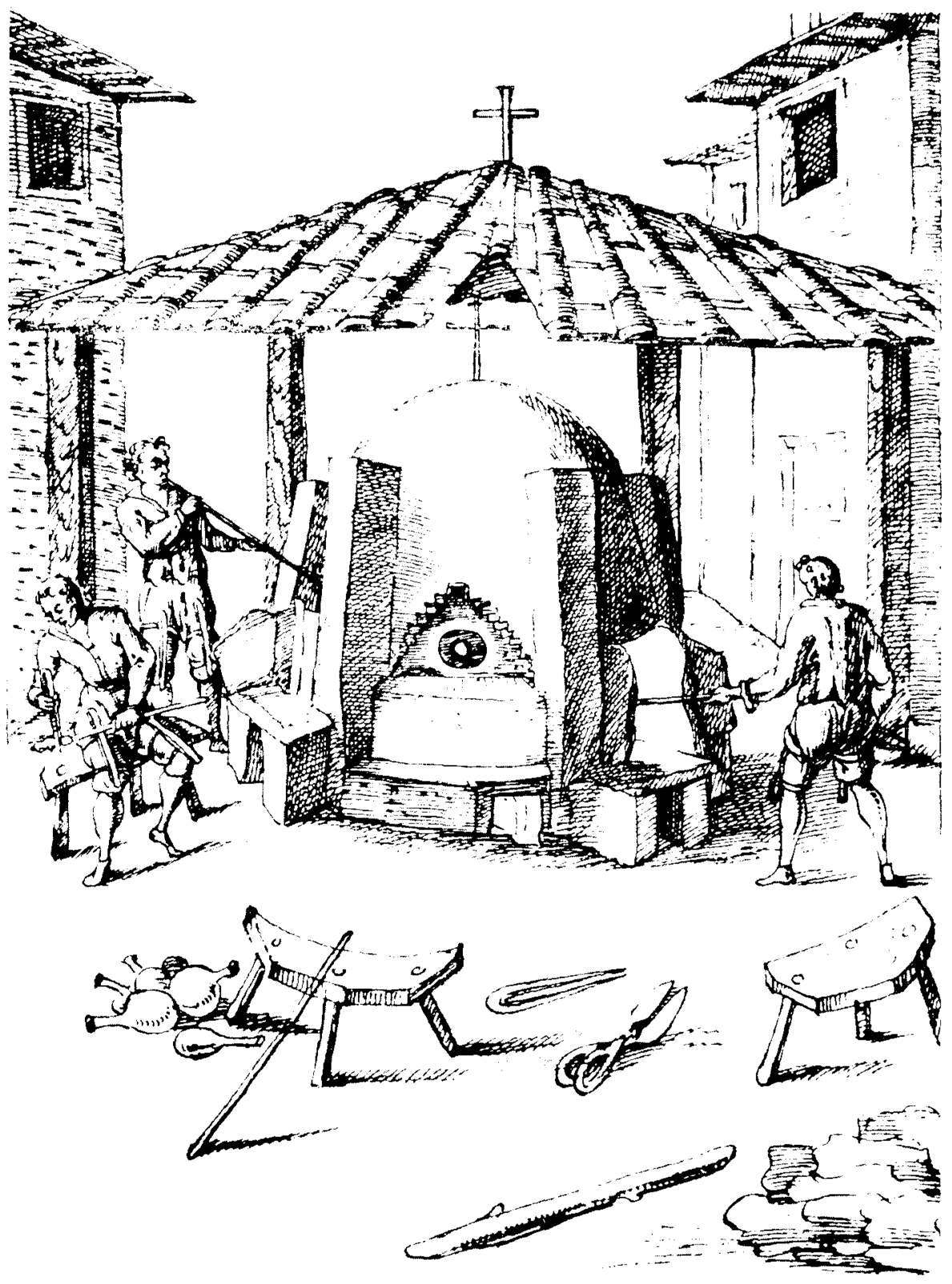

Horno de vidrio. Bichierografia. Giovanni Maggi (1600), 
jetos, venta de los mismos, relaciones entre patrones, maestros y oficiales, distribución del combustible, organización de ferias, festividades, etcétera.

La corporación se regía interiormente por un Consejo elegido cada año por los artesanos, reunidos en asamblea (capitoli), y estaba compuesto por cinco padroni di fornace, uno de los cuales recibia el título de gastaldo maggiore, y por cuatro maestros, dos, con el título de gastaldo della maestranza, y los dos restantes, llamados del comparto, que se encargaban de ir asignando puestos de trabajo a los maestros que pasaban el examen ${ }^{2}$.

La Mariegola establecía reglas de comportamiento moral y religioso, tanto en la vida pública como privada, y, al igual que en todas las asociaciones gremiales, se contemplaban obligaciones sociales de atención a enfermos, viudas, huérfanos...

En 1525, y tras sucesivas revisiones, se elaboró una nueva redacción, con el objeto de poner de relieve el creciente prestigio que el arte del vidrio había adquirido hasta esa fecha, gracias a las innovaciones técnicas incorporadas por los maestros. Los aspectos más destacados fueron los relacionados con la admisión de forasteros en la práctica del arte y la prohibición de salir de la República a los operarios de la isla, disposiciones que obedecian al fuerte proteccionismo estatal ejercido sobre una producción que, dada su gran acogida, llegó a proporcionar a la República una importante fuente de ingresos. Las penas aplicadas a los vidrieros evadidos se fueron incrementando de forma paulatina, a medida que la costumbre se iba extendiendo, llegando en 1597 a ser condenados durante cinco años a pena de galeras $^{3}$.

En Venecia, desde tiempos medievales, los operarios del vidrio estaban organizados en tres categorías laborales: padroni di fornaci, maestri y operai (lavoranti y apprendisti) ${ }^{4}$. El maestro, al igual que en todas las demás artes, era el operario más preparado y sobre el que recaía la responsabilidad de la elaboración de las piezas, así como la aportación

Gaspabeito, A., II vetro di Murano dalle origini ad oggi. Venezia, Neri Pozza Editore. 1958. pág. 64 .

ZECCHIN,, L., “Nuovi appunti di storia vetraria muranese”, Giornale Economico, octubre, enero y marzo, 1957-1958, págs. 18-19.

4 Gasparetto, A., op. cit., págs.50-52. En la actualidad son tres los grados: maestro, servente y garzone, con matizaciones en cada una de las distintas categorías: primo. secondo y terzo maestro, etc. 
creativa e innovadora. Su formación incluía conocimientos de alquimia, que le permitían llevar a cabo investigaciones técnicas relacionadas con su arte. El más directo colaborador del maestro era el operaio lavorante o servente (equivalente al grado de oficial en España), cuya tarea era básica, llegando a veces a sustituirle, dada su buena preparación. Finalmente, estaban los apprendisti o garzoni, los operarios de menor experiencia, cuya labor en el horno solía ser tan elemental como la de cualquier aprendiz de oficio. La edad mínima para ser admitidos eran los 14 años.

Los hornos se mantenían abiertos las veinticuatro horas del día, organizándose turnos de trabajo entre los operarios. Sin embargo, durante cinco o seis meses al año permanecían inactivos, con el fin de ser restaurados o incluso, en caso de necesidad, sustituidos por otros. Esta práctica suponía una pérdida económica para los vidrieros, que consiguieron, a modo de compensación, que el Consiglio dei Dieci les permitiera vender vidrio de forma ambulante durante ese período de tiempo. Es interesante anotar que muchas de las evasiones de gente del oficio tuvieron lugar a lo largo de estos meses de inactividad ${ }^{5}$.

La República de Venecia ejercía un fuerte monopolio sobre la producción de los hornos, pues el Estado adquiría todo el material manufacturado y lo revendía a los comerciantes, que se encargaban de su distribución. Por esta causa, los vidrieros no podían vender sus productos en zonas como Rialto o San Marcos, sin licencia de los Giustizieri, aunque tenian permiso para hacerlo en barrios menos frecuentados ${ }^{6}$.

Muchos maestros del vidrio llegaron a ocupar un puesto destacado en la sociedad veneciana, debido a la gran valoración de su trabajo. Para ejercitar este oficio en la isla era requisito indispensable estar inscrito en el Libro d'oro de la Magnifica Comunità di Murano, privilegio que sólo estaba al alcance de los nacidos allí y de quienes habían obtenido la ciudadanía. El gobierno de la República reconoció la nobiltà del arte del vidrio con respecto a otras artes mecánicas, llegando a ser consicierado "un artesano de elite", en expresión de Rosa Barovier?.

Son muy numerosos los nombres de artífices documentados desde el Quattrocento, pero entre todos ellos destaca la figura de Angelo Barovier

Barovier Mentasti, R. I/ vetro veneziano. Milano, Electa, 1982, pág. 97.

Gasparetto, A., op. cit., pág. 52.

Barovier mentasti, R., op. cit., pág 8. 


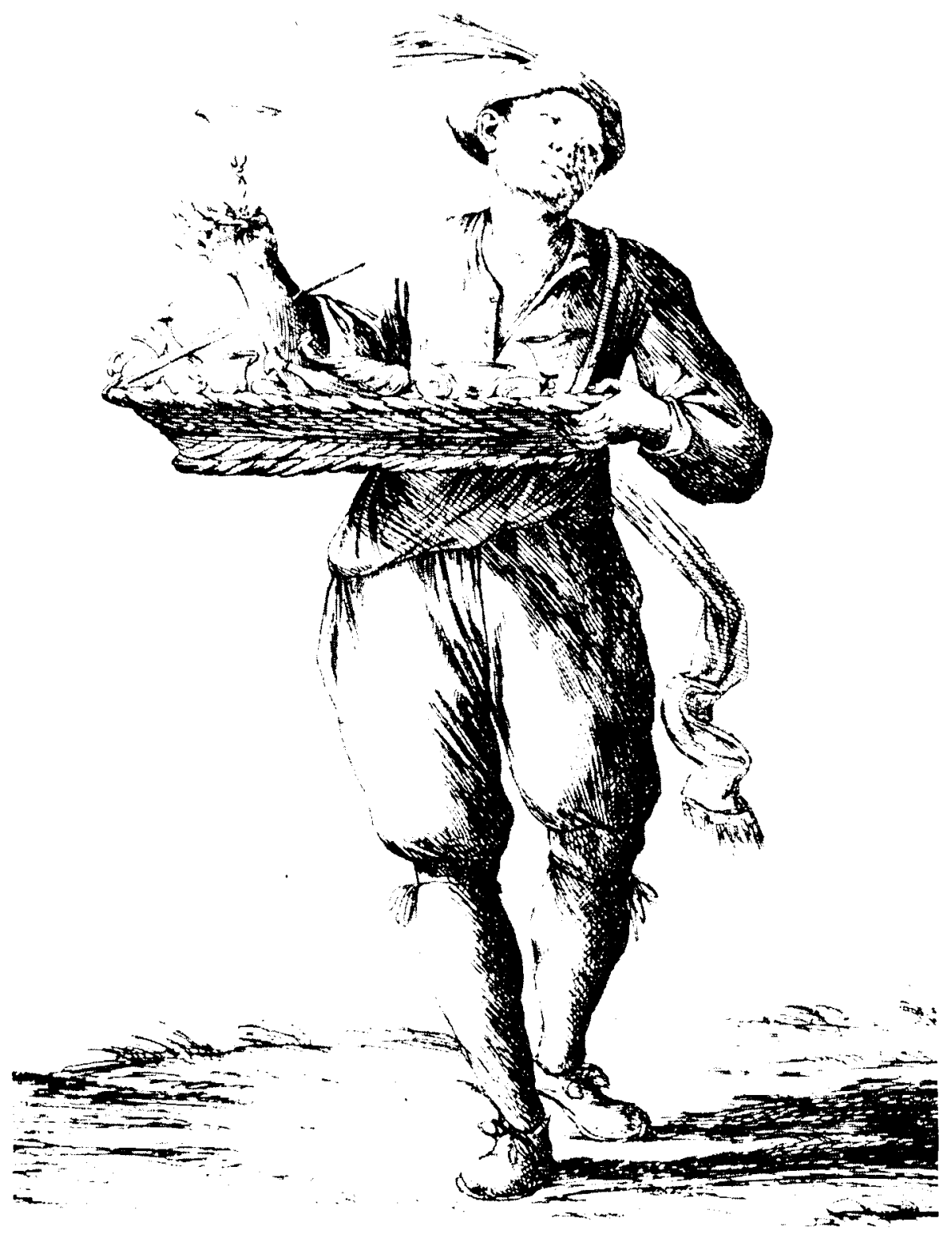

Vendedor de vidrio. Giuseppe Mitelli. Bolonia finales del siglo xVI. Biblioteca Casanatense, Roma. 
$(+1461)$, perteneciente a una familia dedicada a este oficio desde finales del siglo XIII. Su fama le vino dada por la serie de descubrimientos técnicos que consiguió, el más célebre de los cuales, el famoso cristallo (calidad de vidrio caracterizado por su gran ligereza y transparencia), proporcionó fama universal a los hornos de la isla. Asimismo, es considerado como el iniciador de la decoración en vidrio.

Angelo llegó a ocupar cargos importantes, tales como Camarlengo, Cancelliere de la Comunidad de Murano, Lector Apostólico y Secretario papal en Roma. Antonio Averulino, el Filarete, se refiere a él, en su Tratado de Arquitectura, como autor de «belli lavori di Cristalini», y alude a la amistad que les unía a ambos ${ }^{8}$.

Varios miembros de la familia Barovier continuaron trabajando después de su muerte: Marino, al que también cita el Filarete, Giovanni, Francesco y su única hija, María. A comienzos del siglo XVII, un miembro de esta familia, Domenico, "gentilhombre de Murano", vino a España, y se estableció primero en Mallorca y más tarde en El Escorial, en donde trabajó para el rey Felipe III ${ }^{9}$.

Apellidos célebres de familias de vidrieros fueron también los Ballarin, Della Pigna, Mocetto y los Serena, entre otros muchos.

Son, asimismo, muy numerosos los maestros venecianos documentados fuera de la República del Adriático, especialmente en el siglo XVII, centuria en la que se incrementó notablemente el número de evasiones. Entre ellos merecen ser recordados: Antonio Miotti, que trabajó en Bruselas, Francesco y Giovanni Savonetti, Nicola Stua, establecido en Amsterdam; Bernardino Scapitta, que viajó primero a Suecia y más tarde centró su actividad en Inglateria y en Alemania ${ }^{10}$.

Todos ellos, casi sin excepción, formaban parte de estirpes de maestros vidrieros, cuyo negocio, como se tenía por costumbre, pasaba de padres a hijos a lo largo de generaciones. En la actualidad muchas de las grandes firmas siguen llevando los ilustres apellidos del pasado: Barovier, Toso, Seguso...

En contraste con el artista del vidrio de Murano, el vidriero español era un personaje anónimo que trabajaba en su pequeño horno y que atendía

Antonio Averulino Filarete's Tractat über die baukunst. Wien, 1890, pág. 302. en GASPARETTO. A., op. cit, pág. 78.

Para un mejor conocimiento de este maestro y su estancia en España, ver: Rodrigue $z$ Garcia. J.. "Domingo Barovier. vidriero veneciano en España (1600-1608)". Espacio, Tiempo y" Forma, serie IV, $n .94,1989$, págs. $475-500$.

Gaspark ito, A.. op cit., págs. 49-61 


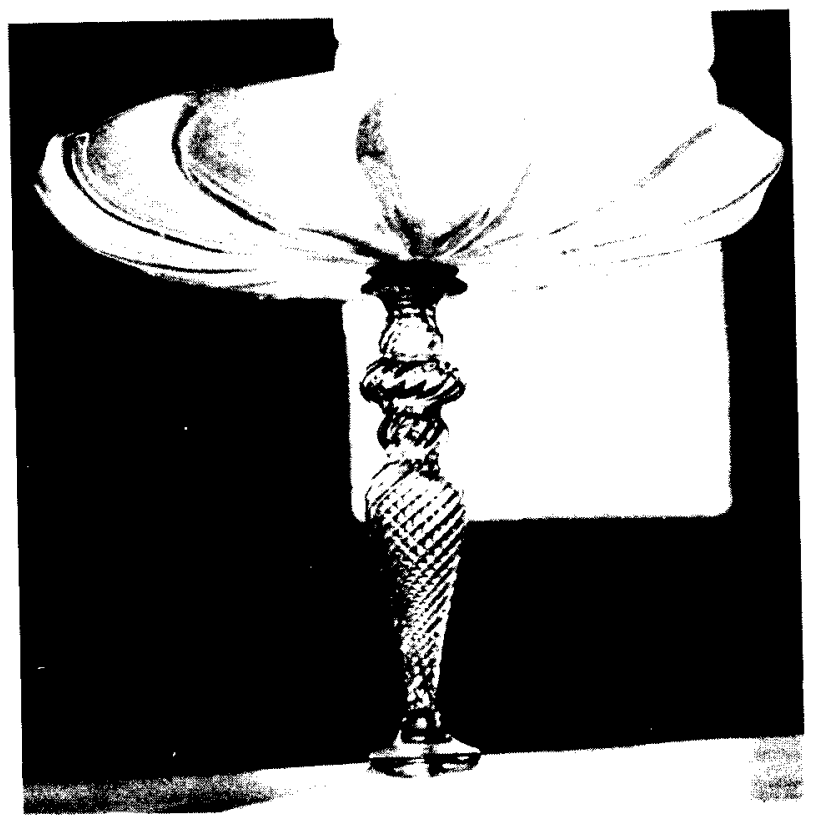

Copa de cristallo veneciana, siglo xvi. Museo Vetrario de Murano.

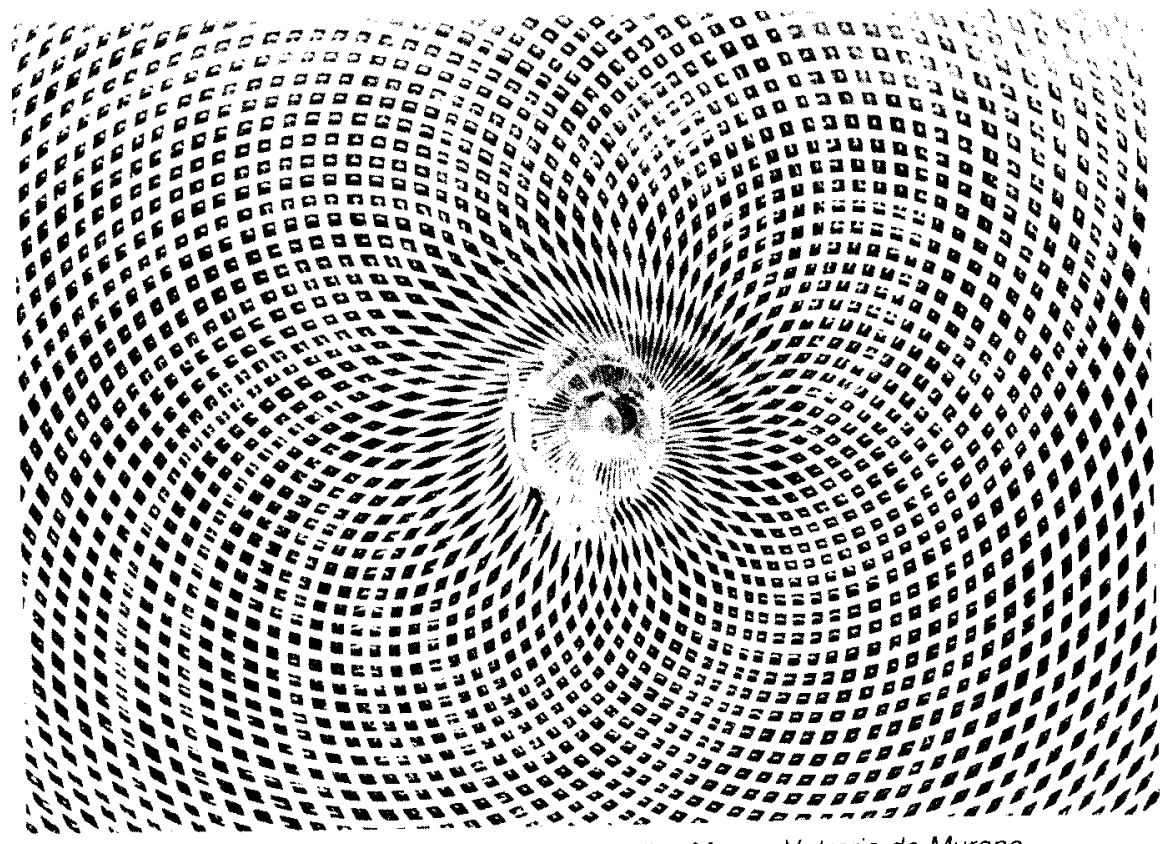

Detalle de un plato de filigrana a reticello. Museo Vetrario de Murano. 
una demanda interior, aunque en algunos lugares, como Barcelona y Cadalso de los Vidrios, se comercializaran sus productos también en el exterior.

La demanda de vidrio veneciano en España -costumbre generalizada en toda Europa, como se ha indicado- fue atendida con la importación de piezas procedentes de Venecia y también de los Países Bajos, en donde trabajaban maestros muraneses muy cualificados. Las manufacturas de vidrio de Murano llegaron a ser un claro signo de status entre las grandes familias de la nobleza, sobre todo en la Corte de Madrid ${ }^{11}$.

Esta circunstancia afectó muy directamente a la actividad de los hornos españoles de las distintas áreas geográficas peninsulares productoras de vidrio, particularmente en aquellas que, por tradición, se manufactuba vidrio intencionadamente artístico, como ocurrió en Castilla y Cataluña. Los vidrieros castellanos y catalanes consiguieron hacer frente a la crisis desencadenada por la dura competencia extranjera, utilizando sus propios recursos para conseguir mantener los hornos activos, aunque, sobre todo en Castilla, se vieron muy seriamente afectados.

Barcelona y Mataró fueron los centros de mayor prestigio en la zona de Cataluña por su especial dedicación a manufacturar objetos suntuarios, muy valorados durante los siglos XVI y xVII. Son muchos los testimonios escritos que acreditan la alta calidad del trabajo de los vidrieros catalanes, así como la presencia de "vidrio de Barcelona», junto al de Venecia, en los inventarios de los bienes de la nobleza española ${ }^{12}$.

De la importancia de estas piezas y de su estimación fuera de España queda constancia en la Bichierografia, de Giovanni Maggi (1606) ${ }^{13}$, en donde

La correspondencia diplomática de los embajadores venecianos con la Corte de Madrid contiene una rica información sobre la entrada de piezas de vidrio de Venecia en España, en es. pecial durante el reinado de Felipe II. El rey tuvo, entre sus múltiples colecciones, una de vidrio de Venecia. Sobre este tema. véase: Ronfigutz Garcin, J., "La Façon de Venise en Castilla". Espacio, Tiempo y Forma, serie IV, n. 8, 1995, págs. 49-61

En muchos inventarios de las grandes familias de la nobleza de los siglos $\times$ vi y $\times$ vil figura, junto a toda clase de bienes suntuarios, una relación de vidrios de Barcelona, además de los de Venecia. El más célebre de estos inventarios es el de Isabel la Católica (1506), en el que se describen 416 piezas de vidrio esmaltado, probablemente de manufactura catalana. Cfr. Guolot Ricart. J., Los vidrios catalanes, Monumenta Cataloniae, vol. III. Barcelona, 1941, págs 45-46.

La Bichierografia, de Giovanni Maggi, es un conjunto de cuatro códices. que se conservan repartidos entre la Biblioteca Nazionale de Florencia y el Gabinete de Dibujos de los Uffizi, con los 


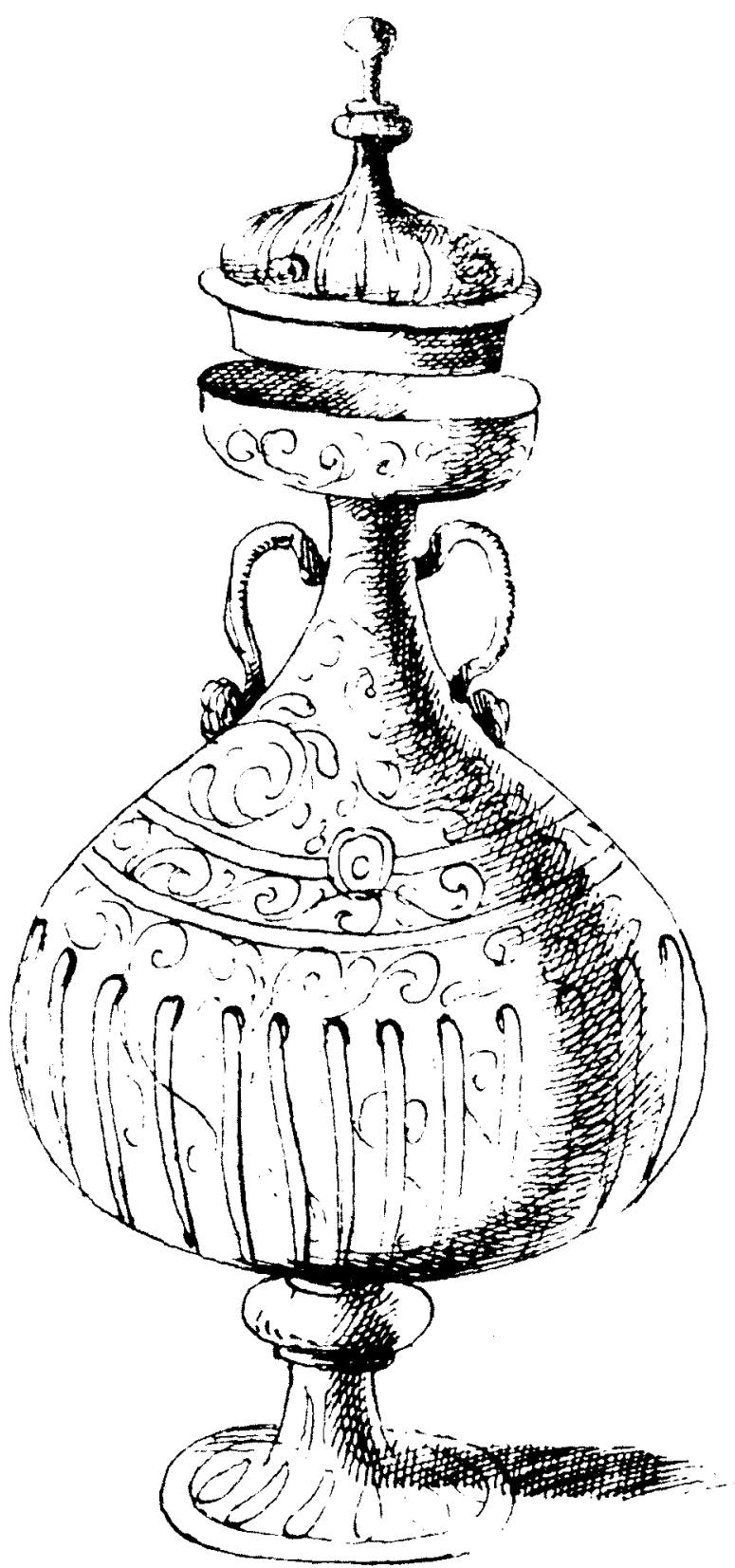

Botella esmaltada catalana en la Bichierografia. Giovanni Maggi (1600). 


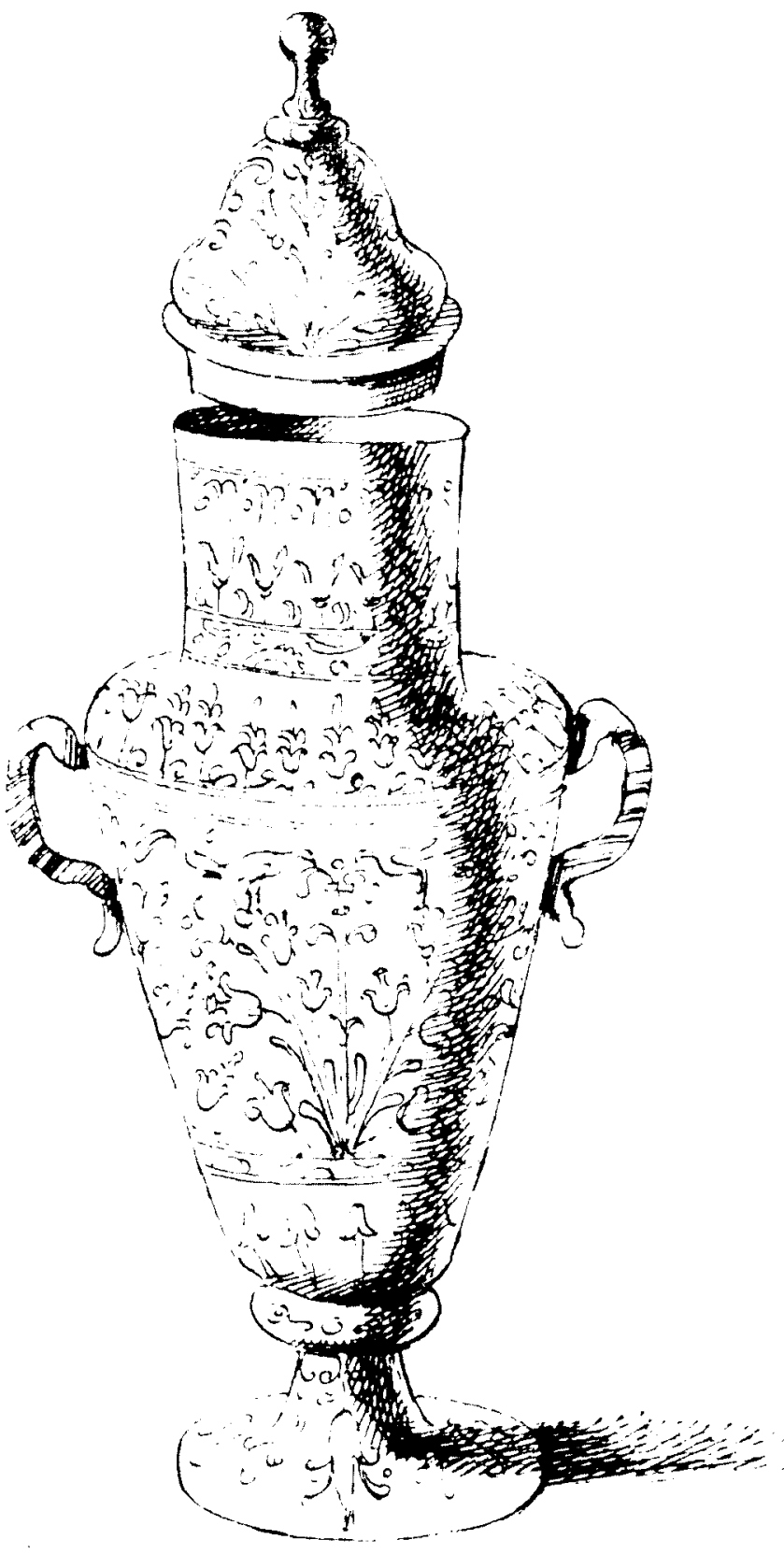

Jarra esmaltada catalana en la Bichierografia. Giovanni Maggi (1600). 
aparecen, entre los 1600 dibujos de objetos de vidrio pertenecientes al Cardenal del Monte, varias piezas de Barcelona, algunas de ellas esmaltadas ${ }^{14}$.

El vidriero catalán ejercía su oficio controlado muy directamente por las asociaciones gremiales. En el siglo $x v$ el gremio de vidrieros estaba unido al de los esparteros, y en 1456 se redactaron sus primeras ordenanzas ${ }^{15}$, las cuales fueron presentadas el 20 de octubre de 1595. En el aspecto laboral se disponía que el operario o aprendiz que trabajase con un determinado maestro no podría cambiar de horno hasta no haber cumplido los cuatro años reglamentarios, excepto por enfermedad o voluntad de éste. Asimismo, los maestros no podían contratar aprendices por menos de cuatro años.

En cuanto a la comercialización del vidrio de horno (así llamado para distinguirlo del de llum o soplete) era necesario haber ejercido cuatro años el oficio y pagar 25 sueldos de entrada en la cofradia para poder abrir una tienda. La venta ambulante sólo estaba permitida a los del gremio o familiares, previo pago de un estipendio. Sin embargo, en las ordenanzas de 1659 , que anulaban las de 1595 , se prohibía a todas las categorias profesionales, incluso a los maestros ${ }^{16}$.

La venta de vidrio de /lum, tanto ambulante como en tienda propia, estaba permitida a todo el que hubiera hecho el aprendizaje de cuatro años y pasado el examen para vidrier de llum (para realizar esta prueba había que pagar una cuota, que se reducia al cincuenta por ciento para los hijos de los cofrades).

Cualquier incumplimiento de las normativas de las ordenanzas se sancionaba con multas, cuyo importe pasaba a un fondo destinado a las obras asistenciales de la cofradía. También se especificaba detalladamente todo lo relativo a las festividades religiosas del santo patrono, que pasó a ser

dibujos, realizados por el pintor Giovanni Maggi. de los objetos de vidrio de la colección del Cardenal del Monte. Esta obra se ha publicado en Florencia (1977) en una bella edición facsimil, a cargo de Paola Barocchi, en dos volúmenes.

Los llamados pitxers en Cataluña, recipientes ovales con perfil achatado, se han considerado habitualmente objetos para contener flores. Sin embargo. gracias a los dibujos de Maggi, se puede comprobar que llevaban tapadera, es decir, que no eran floreros. sino botellas. Todos los pitxers que se conservan en la actualidad han perdido la tapadera.

Para una mayor información sobre este tema, ver: ROURiGuez GARCIA, J., "Vetri artistici catalani nella "Bichierografia" di Giovanni Maggi (1604)", Rivista della Stazione Sperimentale del Vetro Venezia. 199, n. ${ }^{\circ}$ 3, págs. 129-136. fol. $37 \mathrm{v}$.

Archivo Histórico Municipal de Barcelona (AHMB). Registro de Orainacions, 1456-1462,

AHMB, Registro de Ordinacions, 1658-1663, fol. 29 
San Miguel Arcángel -desde que los vidrieros se separaron de los esparteros - y a las actividades de carácter asistencial.

Como en todos los oficios artesanales, la graduación de los operarios tenía tres niveles: maestro, oficial y aprendiz. El examen de maestro se realizaba ante tres personas del Consejo del gremio. El aspirante debía pagar 25 libras al tesorero en concepto de examen y 20 sueldos a cada uno de los examinadores. Caso de ser hijo o yerno de maestro. se percibía una considerable reducción en las tasas.

Las manufacturas de los vidrieros catalanes alcanzaron un buen nivel de calidad, tanto desde el punto de vista estético, como técnico, a juzgar por los numerosos testimonios escritos elogiando la labor de estos artífices y por las piezas que nos han llegado.

La feria del vidrio, que se celebraba en Barcelona el primer día del año, era un importante acontecimiento en la ciudad. La mercancía se exhibia en la plaza del Born y en la calle de la Vidriería, vecina a dicha plaza. El vidrio artístico ocupaba un lugar relevante en la muestra, pero era más abundante el vidrio común, dada su mayor demanda. Como la feria coincidía con el día de Año Nuevo (Ninou) era visitada por los concellers, que salían en cabalgata por la ciudad.

Merece ser recordado por su belleza, el texto que dedicó Tirso de Molina en El Bandolero a dicha muestra, del que vamos a transcribir sólo un fragmento:

"Dos ferias hacen franca a Barcelona cada invierno en la materia más lúcida, más delicada y quebradiza, si bien más útil que halló el uso del hombre. Vidrio es la una, que en el primer día de enero, no contentandose con los hielos de que la distancia del sol le viste, obliga al artificio que adorne su mayor plaza y sus vecinas calles de tiendas cristalinas, que en desahogados aparadores, vajillas, aguamaniles, vasos, escritorios, retablos, sortijas y brinquillos de vidrio transparente hermosean los portales de las casas con algún género de menosprecio de la Argentería (así llama esta ciudad las oficinas de los dos príncipes metales)

Esmérase esta nación, sobre las demás de España, en lo aliñoso y sutil de sus tares (...), y las que en los aseos emulos del cristal emplea Barcelona, compite con Venecia "; pues dado que sus vidrios tengan por extranieros mayor estima, si en la sutileza de su labor deja igualarse, en las diferencias curiosas y confusión apacible de sus hechuras no lo permite: testigo es la experiencia con que por toda nuestra patria guarnece manos, gargantas y cue-

En el texto de Tirso de Molina queda patente la inevitable comparación con los objetos venecianos ( pues dado que sus vidrios tengan por extranjeros mayor estima"), en donde queda muy bien parada la fantasía y creatividad de los artesanos catalanes. 
llos, vestidos, oratorios y camarines, de las dádivas que Barcelona feria a sus damas, para que a imitación de cuanto añade la industria invencionera al valor de los metales, no lo echen de menos, pues a no cederles la frágil duración de su materia, lo diáfano y hermoso de sus vidrios hubieran hecho despreciable el oro" ${ }^{18}$.

La presencia de piezas catalanas en el extranjero se pone de manifiesto no sólo en la Bichierografia, sino también a través de unos inventarios de vidrieros florentinos del siglo XVI, en los que aparecen relacionados objetos de vidrio de Cataluña. En uno de ellos, perteneciente a un tal Lorenzo d'Orlando, vidriero (1546), se incluyen "tre taze alla catalana col nodo grosso, pichole», y más adelante, " 24 taze catalane a nodo groxo» ${ }^{19}$.

En 1540 se vuelve a mencionar en un documento veneciano "chatelani de retortoli chiari, n. $10{ }^{20}$, y, finalmente, en el inventario de Bortolo d'Alvise (1570), vidriero muranés emigrado a Florencia, se incluyen "cathelani a gamba" $y$ "cathelani con filo d'oro" ${ }^{21}$.

Estos objetos, a los que se alude en los citados inventarios, podrían ser importados de Cataluria o manufacturados en Murano siguiendo un modelo autóctono catalán. En cualquier caso es interesante ver que la producción de esta zona de España era conocida y valorada en Italia, siendo muy significativo que pudiera introducirse en un ámbito tan destacado en la manufactura de vidrio suntuario ${ }^{22}$.

A este respecto es oportuno recordar la cita de Lope de Vega en El Abanillo:
"Oí decir en Italia
que de vidrios exquisitos
era rica Barcelona”, 23.

¿Cómo respondieron los vidrieros catalanes a las exigencias de la moda del momento, es decir, a la imposición de los cánones venecianos?

Examinando el amplio muestrario de objetos de vidrio suntuario catalán que nos ha llegado, se puede apreciar indudablemente la influencia de la

TiRso ue Molina, El Bandolero, ed. de A. NOGUÉ. Madrid, 1979, págs. 85-86.

CANTINI GuIDoti, G... "Tre inventari di bicchierai toscani fra Cinque e Seicento", Quaderni della Crusca, 2. Florencia, 1983, pág. 157

ZeCCHIN. L., "ll quaderno del Bertolussi», Rivista della Stazione Sperimentale del Vetro. VI. 1, 1976, pág. 20

ID.. "Bortolo d'Alvise ai tre Mori», Vetro e Silicati. XIII. 1969, pags. 24-25.

Puede servir de dato comparativo al respecto el hecho de que en la actualidad se fabriquen en Murano unos bellos objetos esmaltados, que reproducen, más o menos fielmente, un conocido pitxer catalán del Museo de Murano. Estos objetos se conocen con el nombre de catalane.

Ainaud de Lasakte. J., Cerámica y vidrio, en Ars Hispaniae, vol. X. Madrid. 1952, pág. 346 

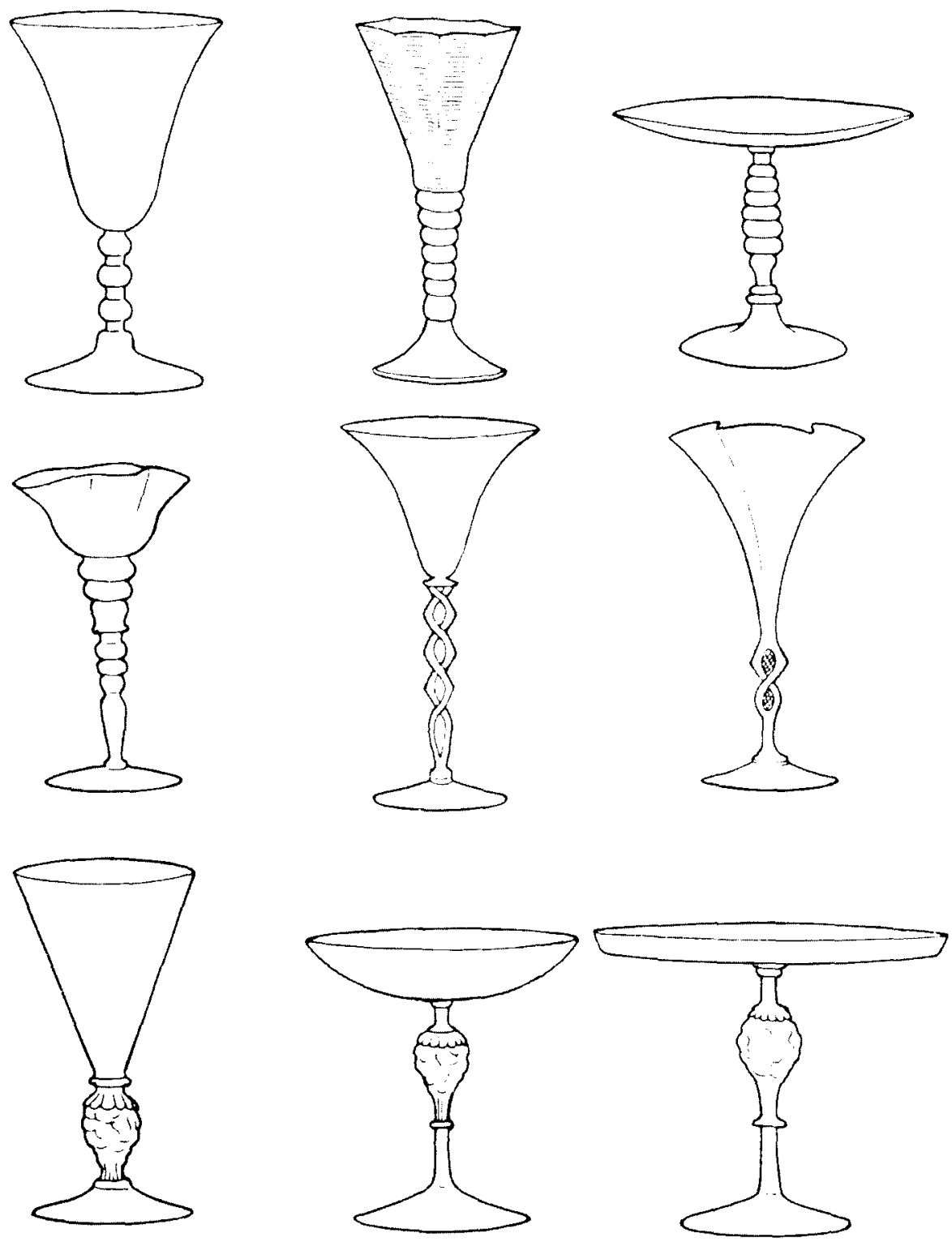

Tipologias de piezas catalanas de influencia veneciana. Siglos XVi-XVII. Departamento de dibujo. UNED. 
obra de los muraneses. Sin embargo, no fueron, como en otros países, maestros venecianos los que trabajaron en Barcelona ${ }^{24}$.

¿Por qué no se optó en Cataluña por la solución de atender la demanda trayendo operarios de Venecia? Evidentemente, por la oposición del gremio de vidrieros, que decidió asumir esta exigencia del mercado y hacer frente a la demanda con su propio esfuerzo y empeño. Los vidrieros catalanes consiguieron imitar buena parte de las técnicas empleadas por los venecianos (el cristallo, el vetro a ghiaccio o craquelado, la decoración grabada al diamante, etc.) y también reproducir las tipologías más comunes de la producción muranesa. Sin embargo, conservaron las formas autóctonas (el porró, la almorratxa y el càntir), decoradas a la veneciana.

Algunas técnicas más cornplicadas, como la filigrana, el vetro calcedonio o los murrini o millefiori, no se llegaron a manufacturar en Cataluña ${ }^{25}$.

Esta producción catalana pudo hacer frente a la demanda interior y, como hemos visto, también estuvo presente fuera de España.

Las piezas más destacadas de las manufacturadas en Barcelona son, sin lugar a dudas, las esmaltadas, de las que se conserva una importante serie, repartida entre colecciones y museos españoles y extranjeros.

A través de la documentación de la época, se pueden conocer algunos datos sobre los artífices del vidrio: lugar en donde trabajaron, contratos de compraventa y arrendamiento de inmuebles para establecer los hornos, inventario de sus bienes, etc. ${ }^{26}$. Sin embargo, ninguna fuente documental, salvo

¿ En Cataluña puede considerarse casi inexistente, a juzgar por la documentación consultada, la presencia de operarios venecianos: he podido documentar 300 vidrieros activos en Cataluña entre los siglos XVI y XVII, la gran mayoria de los cuales tienen apellidos catalanes, algún francés. y sorprendentemente, la ausencia de venecianos o de otras zonas de italia, es casi total (sólo un tal Gaspar Veneciano, que estuvo en Barcelona en torno a 1580;

25 Para un conocimiento pormenorizado de las técnicas venecianas, véase, entre otros muchos trabajos: Barovier MENTASTi, R., I/ vetro veneziano..., op. cit.; véase también la influencia en Cataluña en: Rodriguez Garcí, J., "La influencia del vidrio de Venecia en Cataluña", Annales du 10 Congrés de I'Association Internationale pour / Histoire du Verre. Madrid-Segovia, septiembre, 1985 , págs. $421-432$

26 El Archivo de Protocolos de Barcelona y el de la Catedral de la misma ciudad contienen un interesante fondo documental para la localización de estos artesanos del vidrio. Sus nombres aparecen en contratos, en las Actas de matrimonio, etc., pero por lo general, son escasos los datos que aporta esta documentación a la hora de obtener una información sobre la actividad laboral de estos hombres. Al igual que en Murano, en Cataluña el oficio de vidriero pasaba de padres a hijos: el apellido Sala aparece repetido en numerosos maestros del siglo xV en Vallromanes, y en la centuria siguiente, en Barcelona. Los Tremolet trabajaron en Mataró a finales del xvi y principios del xvil; Roig fue el apellido de otra familia activa en Mataró a comienzos del $x$ VII, entre otros ejemplos. Todos estos datos están tomados del Repertorio de Vidrieros, en Rodriguez Garcia, J., El vidrio veneciano de los siglos xv al xvil y su influencia en Cataluña. Madrid, 1986, t. II, pág. 880 y ss. (Tesis Doctoral inédita). 
las literarias, nos informa sobre la actividad de estos hombres: infraestructura de los hornos, calidad de las piezas, ténicas empleadas... Los datos en este sentido nos los facilitan las piezas que han llegado hasta hoy y los testimonios escritos coetáneos, algunos tan interesantes como el de P. Gil, quien en su Historia Natural de Catalunya (1600) describe minuciosamente las distintas maneras de manufacturar vidrio en tierras catalanas y facilita abundantes explicaciones técnicas ${ }^{27}$, que proporcionan importante información. La historia del vidrio en tierras catalanas, como la de cualquier zona de España, no des-

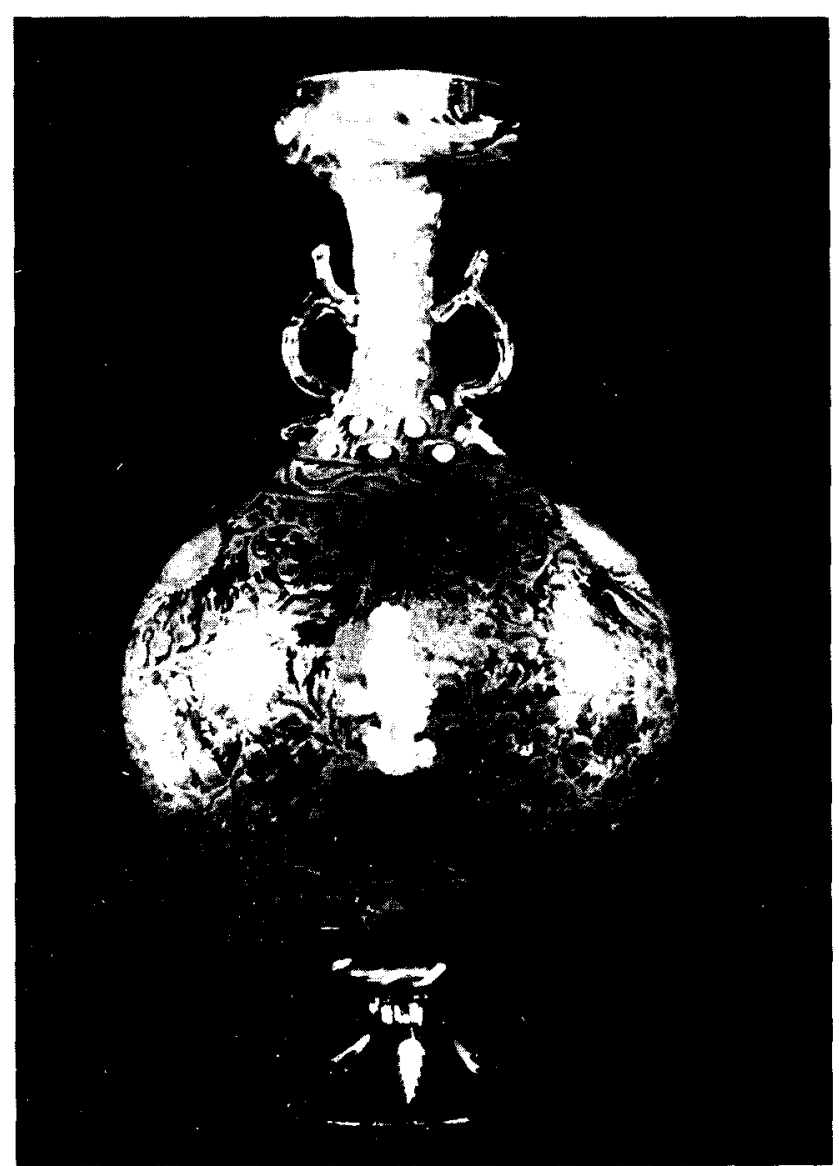

Pitxer esmaltado catalán, siglo xv. Instituto Valencia de Don Juan. Madrid.

P. Gll, Historia Natural de Catalunya, ms. CXXX-5 de la Biblioteca Episcopal del Seminario de Barcelona, publicada por IGl. ESIAS, J. Barcelona, 1949. 


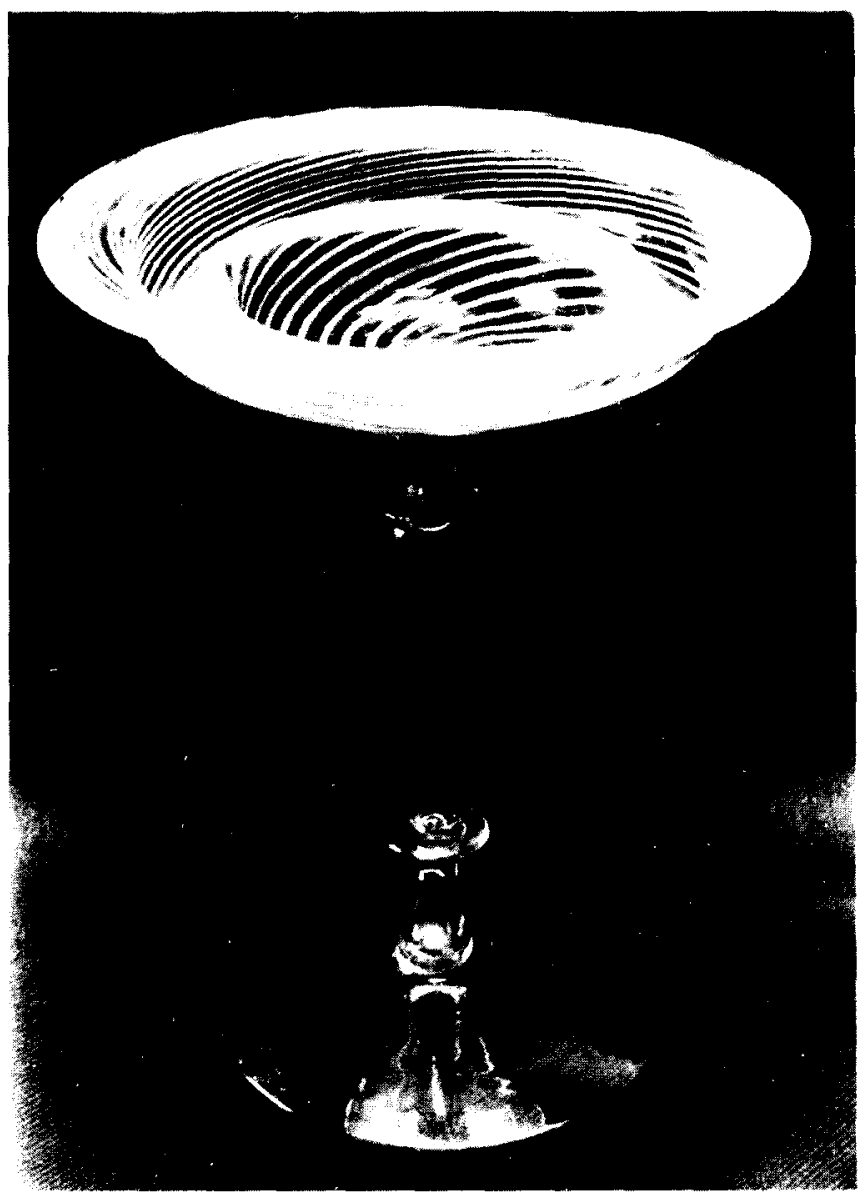

Copa catalana de vidrio cristalino con hilos helicoidales de lattimo, siglo xv. Instituto Valencia de Don Juan, Madrid.

taca por el protagonismo de sus artífices, ni por las aportaciones técnicas, como vimos en Murano. Sin embargo estos artesanos supieron realizar una labor de calidad, avalada por un largo pasado de experiencia y tradición, que les facultó para hacer frente a la demanda social de una producción cualificada y competitiva, sin necesidad de utilizar mano de obra extranjera.

Como contraste, en Castilla, en donde existió durante la Edad Moderna un importante desarrollo de la manufactura de vidrio, actividad favorecida por la vecindad de la Corte y la abundancia de bosques, la competencia foránea, concretamente veneciana y flamenca, fue decisiva para los vidrieros de la zona. Precisamente por la presencia de la 
Corte, Castilla fue muy visitada por artistas extranjeros que se establecieron en Madrid y sus inmediaciones, debido a la atractiva oferta de trabajo que allí se generaba. Los vidrieros castellanos se vieron obligados a competir duramente no sólo con las manufacturas importadas, sino también con los propios artesanos venecianos y flamencos, que, al igual que en otros lugares de Europa, se instalaron en Castilla con el patrocinio real.

A pesar de ello, los centros donde tradicionalmente se manufacturaba vidrio - Valdemaqueda, Cadalso de los Vidrios, San Martín de Valdeiglesias, El Quejigal, Recuenco, etc.- continuaron su actividad durante estas dos centurias, intentando responder adecuadamente a las exigencias del mercado.

En Valdemaqueda, por ejemplo, los hornos estaban todavia activos en 1680 y se trabajaba a la manera de Venecia, dato que conocemos a través de una tarifa de precios de ese año, en la que figuran «copas labradas y otras hechuras particulares de Barcelona, Valdemaqueda y Villafranca, contrahechos de Venecia, a cuatro reales» ${ }^{28}$. En esta localidad se establecieron los maestros Juan Danis y Francisco Herranz, cuyos nombres se conocen por el Tratado que ambos escribieron sobre la obtención de las distintas clases de vidrio y el modo de hacer las vidrieras ${ }^{29}$.

El centro castellano de más renombre fue, sin duda, Cadalso de los Vidrios, aunque se ha investigado muy poco su historia. Una vez más, son los testimonios literarios coetáneos - que ensalzan los productos de sus hornos y la gran difusión que tuvieron - y la presencia en los inventarios de bienes de los "vidrios de Cadalso" los que informan sobre la calidad del trabajo de los artesanos de este lugar. Se tienen noticias de un maestro llamado Juan Rodríguez, activo en la primera mitad del siglo XVI, "maestro de labrar el vidrio de todas suertes, así de lo común, como de lo blanco e verde e de lo rayado a la manera de Venecia", que aprendió su arte en Murano y en Barcelona, y trabajó en Sevilla, Cadalso y Alcalá la Real (Jaén) ${ }^{30}$.

De la producción de Cadalso nos han llegado algunas muestras, pero sorprendentemente no responden al nivel de calidad que debieron tener, si nos atenemos a los documentos coetáneos.

\footnotetext{
¿ Tasa general de alquileres, mercaderias, salarios y jornales de Madrid. Año 1680. Biblioteca Nacional. Varios Especiales, 195

"NiETO AlCAIDE, V., "El Tratado de la tábrica del vidrio, de Juan Danis y el "modo" de hacer vidrieras, de Francisco Herranz", Archivo Español de Arte, n 158, 1967, pág. 298.

30 Gestoso y PÉREZ, J., Ensayo de un diccionario de los artifices que florecieron en esta ciudad de Sevilla desde el siglo x'll al xvm inclusive. Sevilla, 1899-1919, vol. II, pág. 401.
} 
En el reinado de Felipe $\|$ existió un horno en El Quejigal, en donde trabajaron Francisco y Hernando de Espinosa, ayudados por el catalán Gaicerán y Diego Díaz, dedicado a manufacturar vidrieras para el Monasterio de El Escorial, así como objetos de vidrio hueco para el abastecimiento de dicho monasterio, el cual decayó notablemente tras la muerte del monarca ${ }^{31}$.

Pero el fenómeno más interesante en relación con la actividad vidriera castellana es la presencia en la zona de maestros extranjeros. En 1606 llegó a Madrid el veneciano Domenico Barovier, al que ya se ha aludido, procedente de Palma de Mallorca, en donde estuvo cinco años sin llegar a abrirse camino profesionalmente. Este maestro consiguió abrir un horno en El Escorial para manufacturar todas las vidrieras necesarias para la casas reales y sobre todo para el monasterio ${ }^{32}$, con el apoyo real y ayudado por un socio de Ragusa que no era del oficio. La prematura muerte de

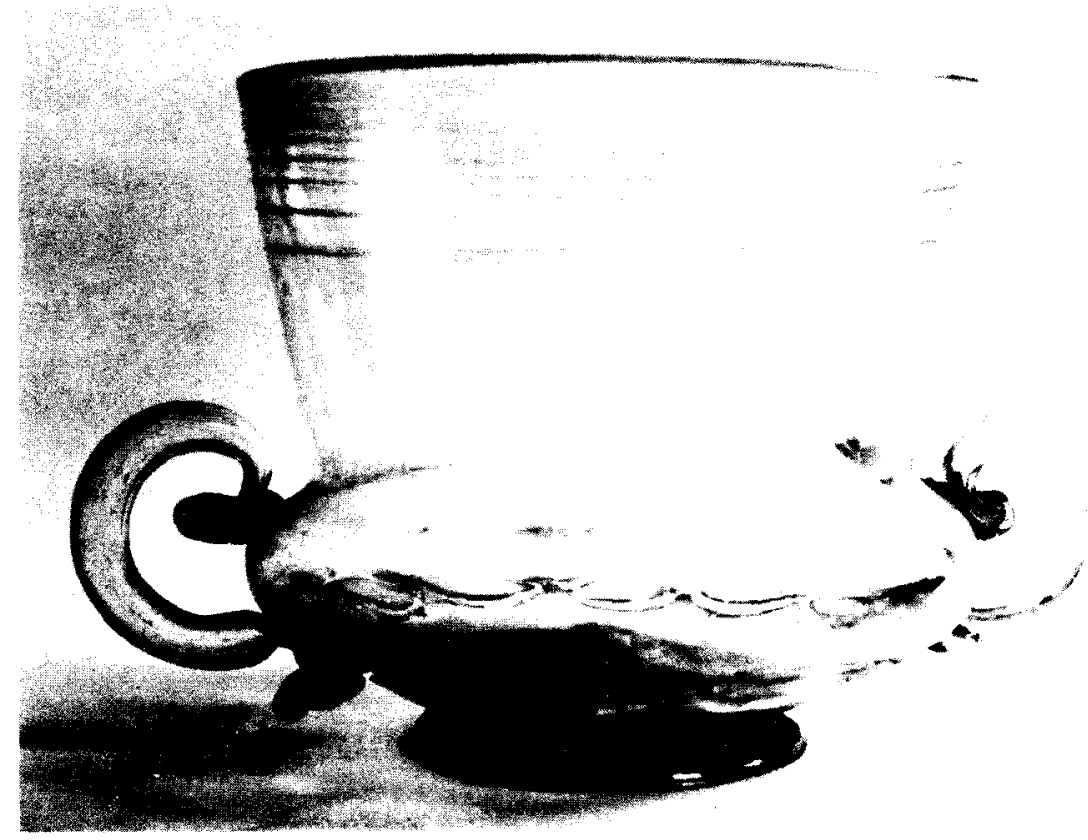

Taza de vidrio incoloro con cadena ornamental aplicada. Castilla (¿Cadalso de los Vidrios?), siglo XVII. Museo Vetrario de Murano.

\footnotetext{
Rıco y Sinobas, M., Del vidrio y sus artifices en España. Madrid, 1873, pág. 39

Para una mayor inforrnación sobre este tema: RodRiGUez GARCiA, J., «Domingo Barovier, vidriero veneciano en España...", op. cit
} 
Barovier acabó con todas las expectativas, aunque su socio intentó sacar a flote el negocio con vidrieros de Cuenca, pero no lo consiguió, ya que éstos no supieron obtener la calidad de vidrio que el rey había concertado en el contrato con el veneciano.

Esta pequeña manufactura en El Escorial reviste un especial interés pues se anticipa en el tiempo, ya que la mayor afluencia de extranjeros al servicio de la Corona tendrá lugar en el último cuarto del siglo XVIl, dentro del marco de recuperación económica del reinado de Carlos II.

En 1679, año en el que se creó en España la Real y General Junta de Comercio, cuyo fin primordial era el fomento de la industria, llegó a Madrid, procedente de Flandes, Dieudonné Lambotte, maestro vidriero, que trabajó en Namur en la fabricación de espejos y vidrios planos. Con el apoyo de la Corona se estableció en San Martín de Valdeiglesias para poner en marcha una manufactura de vidrio suntuario à la Façon de Venise, al servicio de la Casa Real. La envergadura de esta fábrica era de muy distintas proporciones a las de los pequeños hornos artesanales de nuestros vidrieros: Lambotte trajo consigo veinticinco oficiales flamencos y venecianos, mano de obra que consideró necesaria para hacer frente a la producción que se le pedía. Carlos II aprobó las condiciones del contrato en mayo de 1679 , ordenando a la Junta de Comercio los trámites necesarios para su cumplimiento ${ }^{33}$.

El maestro flamenco se comprometió a manufacturar "christal de roca y su fundición para hacer espejos de armar y de todos géneros; vidrios cristalinos para coches, sillas de mano, ventanas y demás usos y para todo género de piezas que se labran según esta sciencia y arte, incluyéndose las piedras coloridas que se engastan..." ${ }^{34}$. A cambio exigía una serie de condiciones - exención de impuestos, monopolio en la manufacturación de estos productos, venta de los mismos libre de alcabala y otras cargas fiscales, especial estatuto jurídico para él y sus acompañantes, etc-

Tres años permaneció la fábrica activa, aunque, al parecer la calidad de los productos no correspondió a lo que el rey esperaba, y el 19 de septiembre de 1683, Lambotte acuerda traspasarla, por motivos de salud, al vidriero Antonio de Ovando, vecino de Cadalso ${ }^{35}$.

\footnotetext{
33 Rodriguez Garcia, J., "Algunas noticias sobre una fábrica de vidrio de Venecia en San Martín de Valdeiglesias (1679-1689)", Espacio, Tiempo y Forma, serie IV, n² 2, 1989, págs. 155-174.

34 Archivo Histórico Nacional (AHN), Consejos, leg. 13-215, 10 de enero de 1679.

35 Ibidem, leg. 7223. Consulta de 19 de septiembre de 1683.
} 
Tras la muerte de Lambotte, la fábrica decayó, intentando levantarla a toda costa el oficial más cualificado que acompañó al vidriero flamenco, Giacomo Bertoletti, de Venecia.

La corta vida de este establecimiento vidriero al servicio de la Corona es un hecho común a todos los intentos de manufacturar en España productos suntuarios con mano de obra extranjera en el último tercio del siglo XVII. Será necesario esperar a la siguiente centuria para que la nueva dinastía borbónica, siguiendo el ejemplo francés, promueva las manufacturas reales con el objeto de proveer a las casas reales de los necesarios objetos suntuarios. Estas fábricas reales estarán también en manos de operarios extranjeros, más experimentados y preparados que los españoles, pero sus resultados económicos tampoco llegaron a ser satisfactorios. 